\title{
Aging Detection Capability for Switch-Mode Power Converters
}

\author{
Jaspreet Kaur Mann, Member, IEEE, Suresh Perinpanayagam, Member, IEEE, and Ian Jennions
}

\begin{abstract}
The detection of degradations and resulting failures in electronic components/systems is of paramount importance for complex industrial applications including nuclear power reactors, aerospace, automotive, and space applications. There is an increasing acceptance of the importance of detection of failures and degradations in electronic components and of the prospect of system-level health monitoring to make a key contribution to detecting and predicting any impending failures. This paper describes a Parametric System Identification based healthmonitoring method for detecting aging degradations of passive components in switch-mode power converters (SMPC). A NonParametric system response is identified by perturbing the system with an optimized multi-tone sinusoidal signal of the order of $\mathrm{mVs}$. The parametric system model is estimated from nonparametric system response using recursive weighted least square algorithm. Finally, the power-stage component values, including their parasitics, are extracted from numerator and denominator coefficients based on the assumed Laplace system model. These extracted component values provide direct diagnostic information of any degradation or anomalies in the components and the system. A proof of concept is initially verified on a simple point-of-load (POL) converter but the same methodology can be applied to other topologies of SMPC.
\end{abstract}

Index Terms - Aging, Digital Control, Parametric Estimation, Switched Mode Power Supplies.

\section{INTRODUCTION}

$\mathrm{T}$ he detection of degradations and resulting failures in electronic components/systems is of paramount importance for industrial applications, including nuclear power reactors [1], aerospace [2-3], automotive [4], and space applications. From an economic perspective, it is recognized that with the use of complicated electronic industrial systems, every product manufacturer wants to increase the lifetime of their product with the objective of reducing unscheduled maintenance, inservice costs and improving the availability of their product [5]. More importantly, addressing the issues of how to detect, diagnose, and predict degradation and failures has been identified as a potential requirement by many industrial organizations [6].

In recent years, the high current and increased performance

Manuscript received July 30, 2015; revised November 13, 2015, Decembe 20, 2015 and January 14, 2016; accepted February 4, 2016.

J. K. Mann, S. Perinpanayagam, and I. K. Jennions are with IVHM Centre, Cranfield University, United Kingdom (e-mail: j.mann@cranfield.ac.uk; suresh.nayagam@cranfield.ac.uk; i.jennions@cranfield.ac.uk). demands of highly integrated Programmable Logic Devices (PLD) have led to miniaturization of these devices down to nanometer and sub-micron scale [7]. Consequently, these advancements in the semiconductor industry have required that the input voltage of these devices be reduced from $5 \mathrm{~V}$ and $3.3 \mathrm{~V}$ to below $3.3 \mathrm{~V}$. These technological advances in PLD and networking microprocessors empowered the power supply industry to advance from a basic four output voltage $(12.0 \mathrm{~V}$, $9.0 \mathrm{~V}, 5.0 \mathrm{~V}, 3.3 \mathrm{~V}) \mathrm{DC}-\mathrm{DC}$ power converter to more than twenty output voltage $(3.3 \mathrm{~V}, 2.5 \mathrm{~V}, 1.8 \mathrm{~V}, 1.5 \mathrm{~V}, 1.2 \mathrm{~V}, 1.0 \mathrm{~V}, 0.9 \mathrm{~V}, 0.8 \mathrm{~V}$ etc.) converters [8]. This led to the use of POL converters in distributed power architecture to minimize power losses and increase efficiency. The tolerance of these low-voltage power supplies is dependent on the load. In traditional power supplies, the tolerance requirement on a typical low-voltage power converter is $\pm 5 \%$. However, with the advent of sub-micron technology as mentioned, the tight tolerance requirement on low-voltage converters has been tightened to $\pm 3 \%$ [9]. This prerequisite inadvertently results in increased stress on the power converter and contributes towards degradation of the components and failure of the system.

The majority of power supply failures aggregate from degradations and failures in the capacitor $C$ [10]. An electrolytic capacitor is a passive electronic component, which degrades and fails significantly in power converters [11-13]. The Equivalent Series Resistance (ESR) of the electrolytic capacitor is a prominent precursor to degradation that provides knowledge of anomalies in the capacitor and the overall performance of the power converter. Now, the question arises as to how these degradations in the capacitor and the overall system performance can be detected through global system parameters. The aging or degradation detection in electrolytic capacitors has been studied using on-line and off-line techniques. The off-line technique interrupts normal operation of the circuit, however, is accurate and simpler to implement [14-16]. The on-line detection monitors aging performance while the circuit is in operation. In [12], ESR deterioration has been extrapolated by measuring input current and output voltage ripple in time domain. Similarly, [13] also use output voltage ripple and [17] propose capacitor voltage measurement to estimate the ESR and hence, capacitor degradation. However, the above method does not provide understanding of anomalies in the entire system.

In the frequency domain, the control-to-output transfer function of the power converter describes the dynamic behavior of the system/power converter [18]. In the transfer function or system response of a basic DC-DC POL converter, the - 
$20 \mathrm{~dB} /$ decade asymptote provides knowledge of the inductor and the output filter capacitor. Similarly, the $-40 \mathrm{~dB} / \mathrm{dec}$ de asymptote reveals information about the ESR of the output filter capacitor. This knowledge of the power-stage network ensures that the degradations and failures of power-stage network elements (inductor and capacitors) can be detected, diagnosed, and prognosticated from the control-to-output transfer function response. From a system-level failure perspective, degradation in any of the power supply components due to uncertainties in the component's tolerances or aging of components with time will affect the performance of the entire electronic system. Therefore, this research aims to study and implement systemlevel health monitoring of DC-DC power converters to detect anomalies in discrete components and mitigate long-term failures in the operation of power supplies.

\section{SySTEM IDENTIFICATION TECHNIQUES}

System identification is an interdisciplinary practice used to characterize the behavior of a dynamic system, usually in the form of a mathematical model [19]. Parametric and nonparametric methods outline two different identification techniques. The user excites/perturbs the system with a deterministic or random excitation signal to achieve nonparametric identification of the system [20]. A possible source of excitation in the system is the inherent noise of the system, such as analogue noise. This ambient system noise is best for real-time health monitoring as it eliminates the impact of initial conditions (time-domain) and leakage errors (frequencydomain), and reduces the burden of designing external perturbation. Nevertheless, different load conditions affect the behavior of the inherent noise and lead to low Signal-to-Noise Ratio (SNR) of the extracted system response. Therefore, researchers prefer external perturbation signals, such as an impulse signal, chirp, pink noise, random noise, and single sinusoid.

The general class of perturbation signals, for instance, DiracDelta with a single pulse amplitude spectrum, is not suitable to measure the response of the system across the pass-band of the system between $10 \mathrm{~Hz}$ and $200 \mathrm{kHz}$. This is because the power of a single pulse at high frequencies close to the ESR $r_{C}$ frequency $F_{E S R}=1 / 2 \pi C r_{C}$ is not high enough to measure the output signal and will result in poor SNR. The high-frequency measurement is essential because the zero introduced by the ESR of the output filter capacitor lies at the high-frequency region of the converter response between the cut-off frequency of the output filter $F_{O F}=1 / 2 \pi \sqrt{L C}$ ( $\mathrm{L}$ is the inductance of the output filter) and the switching frequency $F_{S W}$. Therefore, accurate measurement of the attenuation and the phase of the system requires the frequency sweep to excite the pass-band of the system.

The unity crest factor of Pseudo Random Binary Sequence (PRBS) used by Roinila et al. [21], Shirazi et al. [22], Miao et al. [23] and Barkley and Santi [24], which is identical to the crest factor of the delta function, is ideal for extracting the signal buried in the system noise. However, the amplitude spectrum of PRBS for the Band-Limited (BL) inter-sample assumption decreases inversely with the frequency, limiting the signal-extracting capability up to a certain frequency of the entire spectrum. Moreover, Pintelon and Schoukens [25] corroborate that the amplitude spectrum for increasing the length of the PRBS sequence decreases with frequency and hence, is undesirable for extracting signal information at highfrequency spectra of the power converter. The use of a single frequency sinusoid signal incorporated in the Frequency Response Analyzer (FRA) and described by Gonzalez-Espin et al. [26] accurately measures the system response, but it consumes a significant amount of time to measure the system response. This is the reason why the majority of general excitation signals, such as pseudo-random white noise, impulse, and Dirac-Delta, is rejected because the amount of power available in the signal at high frequencies is not high enough to accurately measure the gain and phase of the system. Therefore, none of the above excitation signals is appropriate in this study.

The present research proposes a multi-tone sinusoid signal as an energy-rich excitation to extract a closed-loop frequency response of the power converter. A multi-tone sinusoidal is a periodic, deterministic broadband excitation with full flexibility to define the amplitude spectrum and frequency resolution. It enables optimizing the amplitude spectrum for desired frequencies of interest before performing any measurements, saving significant post-processing and computational time. Recently, a similar phase shifted excitation has been proposed. However, the proposed method uses only ten frequencies to perform non-parametric system response identification across wide frequency range $10 \mathrm{~Hz}-100 \mathrm{kHz}$, compared to $30 \mathrm{kHz}$ to $80 \mathrm{kHz}$ specified in [27].

From a signal-processing perspective, excitation of the system with inadequate energy in the perturbation signal will provide a low SNR or high uncertainty of the measurements. Therefore, based on the required SNR, the optimized multi-tone sinusoid perturbation excites the system at the required frequencies of interest. The system identification of such a system, wherein knowledge of both high and low frequency dynamics is required, is not only complex but also challenging.

Moreover, the research employs the concept of synchronous In-phase and Quadrature-phase (IQ) demodulation used in radio-frequency communication for frequency response measurements. The non-parametric estimate of the system model using quadrature demodulation extracts even the smallest amplitude of the in-phase and the quadrature phase signal and provides an initial estimate of the characteristics, complexity, and the order of the system.

Once the non-parametric model of the system is available, different model estimators, such as least mean squares and recursive least square [19], [28], recursive Dichotomous Coordinate Descent (DCD)-Infinite Impulse Response IIR adaptive filter [29], can be used to estimate a parametric model of the system. In parametric identification, the user assumes the system model, such as a black-box model, a grey-box model, or a transfer function model, and refines this model from the nonparametric measurements.

Since, the digital control systems have been used to accomplish the majority of the system identification process, 
the characteristic of the perturbation signal between the consecutive sampling instants, i.e. inter-sample behavior largely influences the selection of the identification model [30]. The researchers across the system identification community formulate two assumptions: Zero-Order Hold ( $\mathrm{ZOH})$ and BandLimited. The $\mathrm{ZOH}$ criterion assumes that the sampled signal is constant between the consecutive samples. The spectra of the reconstructed signal, Sinc function, generate dominant frequency component at the sampling frequency $F_{S P}$ along with its harmonics at the higher frequencies $2 F_{S P}, 3 F_{S P} \ldots$ and so on. When the inter-sample behavior of the sampled signal is bandlimited, the power spectrum of the reconstructed signal is zero above a frequency $\omega_{\max }$ (usually half the sampling frequency). Mathematically, when $\Phi(\omega)=0$ for $\forall|\omega|>\omega_{\max }$ the spectra of the reconstructed band-limited signal only produce a fundamental sampling frequency without any harmonics.

The research on system identification of power converters assumes $\mathrm{ZOH}$ as the characteristic of the perturbation signal [23], [31], [32], and uses discrete time models to identify the system model. Since the reconstructed signal is not the exact replica of the signal itself, the $\mathrm{ZOH}$ assumption of the signal can introduce errors and may not be suitable for detecting anomalies in the system. On the other hand, the band-limited assumption of the signal ensures that an exact replica of the signal is reconstructed and enables identifying the continuoustime model in frequency domain. Therefore, in this paper, a continuous-time system model in the form of a Laplace rational fraction is assumed.

Recently, several data-driven frameworks have gained attention in fault diagnosis and model extraction. This includes statistical and non-statistical analysis based methods. For instance, Weighted Least Square, Partial Least Square (PLS) [33-36], total projection to latent structures (T-PLS) [37-38], Principal Component Analysis (PCA) [39-41], Independent Component Analysis (ICA) [42] etc. are some of the statistical methods which utilize input and output data to diagnose faults in the system.

Neural Networks (NN), on the other hand, describe nonstatistical data-driven tool that has been extensively used in industrial applications for fault diagnosis. $\mathrm{NN}$ based tools can be further categorized into supervised and unsupervised based learning. In supervised learning, the data set is labelled as healthy or faulty and the algorithms learn from variations among the labelled data. The data set in unsupervised learning does not include any labelled data, instead the algorithm finds clusters from its own data. Well-established algorithms such as Fuzzy Logic [43], support vector machine (SVM) [44-45], Kalman Filter [46-47] etc. are some of the learning methodologies that assist in non-statistical fault diagnosis.

A weighted recursive least square algorithm that minimizes relative error is used to extract the system model in frequency domain. The power-stage component values, including their parasitics are then extracted from the numerator and denominator coefficients based on an equivalent Laplace model. This extraction requires the knowledge of the output load, which implies the knowledge of the output (or the input) current. These extracted component values allow drift/aging

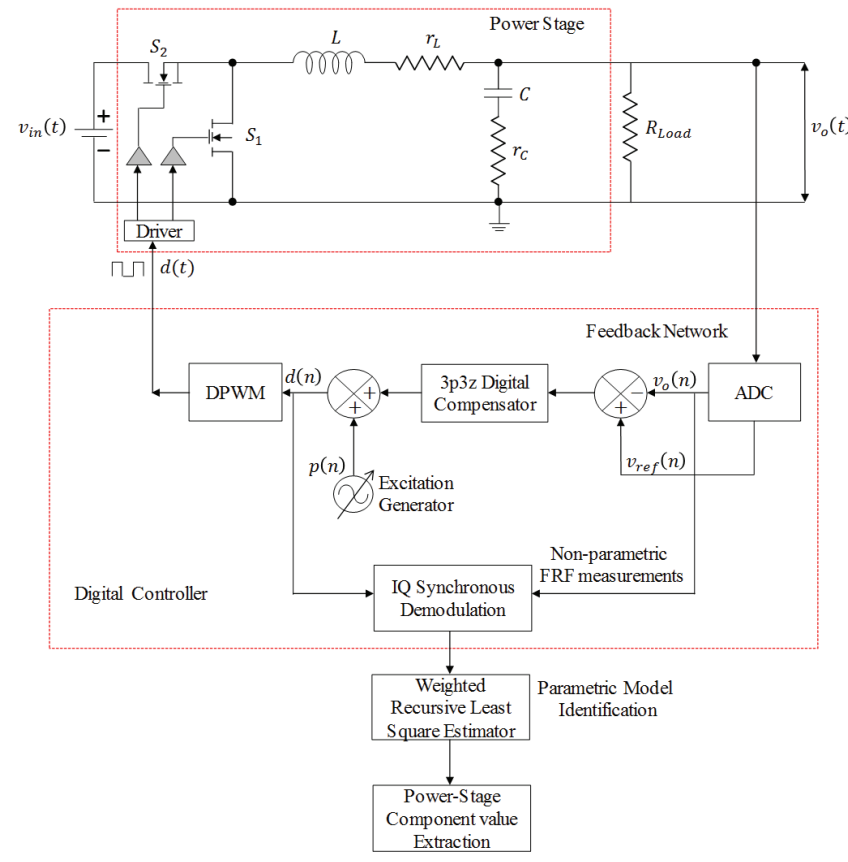

Fig. 1. Block Diagram of Parametric Identification Based Health-Monitoring on Digitally-Controlled DC-DC Buck Converter

measurement of relevant component electrical characteristics and permit diagnostics and end-of-life prognostics.

\section{PARAMETRIC IDENTIFICATION OF DC-DC CONVERTER IN FREQUENCY DOMAIN}

The proposed system identification based health-monitoring methodology is executed on a basic DC-DC buck converter, as shown in Fig.1.

The block diagram includes the power-stage network or the DC-DC power converter regulated by a digital controller in the feedback network. In digital control of power converters using either a Digital Signal Processor (DSP) or a Field Programmable Gate Array (FPGA), the Analog to Digital Converter (ADC) discretizes the output voltage signal $v_{o}(t)$ into a sequence of $\mathrm{n}$ samples $v_{o}(n)$ and computes the error between the digital reference and the digital signal. The digital corrector, usually a Proportional-Integral-Derivative (PID) controller or $3 \mathrm{p} 3 \mathrm{z}$ digital filter, compensates the error to provide a digital sequence $d(n)$. The Digital Pulse-Width Modulator (DPWM) then commands a Pulse Width Modulation (PWM) duty signal $d(t)$ to regulate the control loop. The perturbation signal, which is digitized in the digital controller $p(n)$, excites the control loop and provides a small-signal AC response of the system at the injected frequency of the perturbation. It is worth mentioning that the control loop is not broken and the frequency response is measured in a closed-loop condition.

\section{A. Design of Perturbation Signal}

Ideally, the frequency sweep at every integer frequency up to half the switching frequency provides accurate frequency response measurement. This is similar to the operation of an FRA. However, the memory and computational power of the 
digital controller limits the feasibility of this approach. Instead, the equally spaced frequency sweep of ten or eleven integer frequencies provides maximum system information at the frequencies of interest without restricting the computational power consumption of the digital controller. This multi-tone frequency sweep excites the system at the user-defined frequencies and minimizes power leakage in the adjacent harmonics. Therefore, frequency response measurements by perturbing and linearizing the system with a user-defined frequency sweep provide better insight of anomalies and degradation of the system. More importantly, the selection of integer frequencies close to the ESR frequency and the cut-off frequency of the power converter enable extracting and understanding the degradation of the ESR and the capacitance of the output filter capacitor. Prior to the selection of the perturbation frequencies, periodic perturbation is considered as it reduces measurement noise and enables the spectrum of the signal measured over an integer number of periods to be calculated precisely by the Discrete Fourier Transform (DFT).

The perturbation frequency sweeps logarithmically by octaves from the start to the end frequency to generate a multitone signal. Mathematically, the frequency sweep $F_{P}$ is defined by

$\mathrm{F}_{P_{m}}=\frac{\mathrm{F}_{\mathrm{S}}}{1.2^{\mathrm{m}}}, \mathrm{m}=2,3, \ldots 12 ; \mathrm{l}=1$

where $m$ and $l$ are integers. $l$ can be modified to values 3,5 , or 7 to generate different frequency sweeps. The selection of $l$ generates different logarithmic sweeps by octaves. Using (1), $F_{S}=200 \mathrm{kHz}$ and $l=1$, the start and end frequency tones can be calculated. This equates to different frequency sweeps as shown in Table I. For $l=7$, eleven tones from $85.44922 \mathrm{~Hz}$ to $87500 \mathrm{~Hz}$ excite the pass band of the converter (zero until half the switching frequency). Similarly, for $l=5$, eleven tones from $61.035 \mathrm{~Hz}$ to $62500 \mathrm{~Hz}$ can describe the entire response of the system, including the ESR frequency. For lower values of $l$, more frequency sweeps are available, for instance, twelve for $l=3$ and thirteen for $l=1$.

However, the intention is firstly to excite the system with the minimum number of sweeps to reduce measurement time and secondly to sweep only the frequencies that add knowledge to health monitoring. This results using the frequency sweeps either for $l=3,5,7$. Nevertheless, the sweep frequencies are far apart for the above cases, as shown in Fig. 2.

For $l=1$, on the contrary, 13 frequency sweeps can be reduced by eliminating the lower frequencies, such as $12.207 \mathrm{~Hz}$, $24.414 \mathrm{~Hz}$, and $48.828 \mathrm{~Hz}$ as they do not provide significant

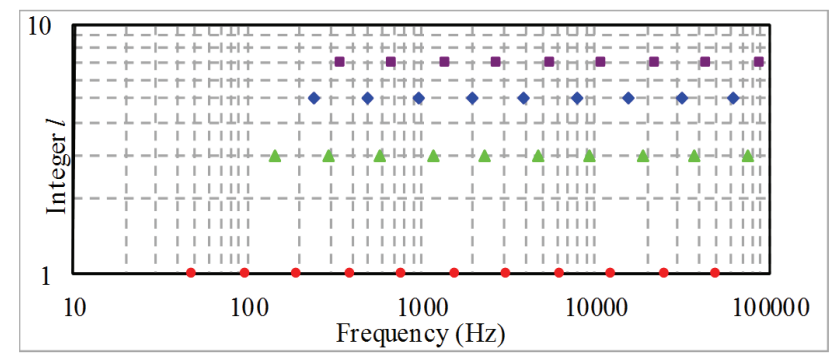

Fig. 2. Comb Spectrum for $l=1,3,5$, and 7 system information for this application. Therefore, for $l=1$ and a power converter switching at $200 \mathrm{kHz}$, a maximum of ten equidistant discrete tones once every octave provides extensive information about the entire system response. This frequency scaling is essential because it enables proper representation of the power-stage frequency response and it includes the powerstage cut-off frequency and the zero introduced by $r_{C}$. This comb spectrum injects the maximum energy at the specified frequency and eliminates spectral spurs that originate at frequencies other than the frequency associated with the discrete tones.

The amplitude of the perturbation signal must be greater than ambient system noise to measure the system response precisely but small enough to keep the system linear and stable. Moreover, the amplitude of the perturbation signal governs the SNR of the non-parametric frequency response measurements and hence, the SNR of the system response. Therefore, to achieve a desired SNR at all the frequencies of interest, it is necessary to optimize the amplitude of the perturbation with respect to the gain of the power converter. In order to achieve a given SNR and minimum uncertainty on the measured system response, different aspects of the digital controller and the power converter need to be analyzed.

The control-to-output characteristics of the POL converter [17] signify that the amplitude of the output voltage is high at lower frequencies compared to the duty signal. Correspondingly, the amplitude of the duty signal is high at higher frequencies, compared to the output voltage. From the above analysis and assuming the presence of additive white Gaussian noise introduced by samplers, it is not feasible to maintain a constant SNR across the frequency spectrum by injecting a constant amplitude perturbation. This would mean injecting large signals at high frequencies leading to saturation of the duty signal.

In addition, it follows that variations/ripple on the output voltage can be measured up to a certain frequency, typically crossover frequency $F_{X}=\left(1 / 20^{t h}\right), F_{S}=10 \mathrm{kHz}$ and two more octaves. However, for higher frequencies, typically $25 \mathrm{kHz}$ and $50 \mathrm{kHz}$, the control loop has to be disturbed to measure a significant amount of variation of output voltage from the ambient noise while maintaining the SNR. For instance, assuming the entire loop noise is due to ADC

TABLE I

SELECTION OF FREQUENCY SWEEP BASED ON THE VALUE OF $l$

\begin{tabular}{crrrr}
\hline \multicolumn{5}{c}{ Perturbation Frequency } \\
\hline$m$ & $l=1$ & $l=3$ & $l=5$ & $l=7$ \\
2 & 50000 & 150000 & 250000 & 350000 \\
3 & 25000 & 75000 & 125000 & 175000 \\
4 & 12500 & 37500 & 62500 & 87500 \\
5 & 6250 & 18750 & 31250 & 43750 \\
6 & 3125 & 9375 & 15625 & 21875 \\
7 & 1562.5 & 4687.5 & 7812.5 & 10937.5 \\
8 & 781.25 & 2343.75 & 3906.25 & 5468.75 \\
9 & 390.625 & 1171.875 & 1953.125 & 2734.375 \\
10 & 195.3125 & 585.9375 & 976.5625 & 1367.188 \\
11 & 97.65625 & 292.9688 & 488.2813 & 683.5938 \\
12 & 48.82813 & 146.4844 & 244.1406 & 341.7969 \\
13 & 24.41406 & 73.24219 & 122.0703 & 170.8984 \\
14 & 12.20703 & 36.62109 & 61.03516 & 85.44922 \\
\hline
\end{tabular}


quantization noise, injecting more than $100 \mathrm{mV}$ of perturbation amplitude would clamp the duty signal. This dictates that to achieve a given SNR at higher frequencies, averaging a large number of measurements is essential. Consequently, if the SNR across the band of frequencies is not constant compared to the limited amplitude of the perturbation, which is certainly the case for frequencies greater than the crossover frequency, averaging over large measurements will yield a given SNR. This would certainly increase the measurement time at the expense of a constant SNR across the frequency spectrum.

Moreover, the quantization noise introduced during analogue to digital conversion diminishes the SNR on the transfer function. This can be resolved by using a high-bit ADC. However, if this not feasible, it must be guaranteed that the amplitude of the perturbation signal is greater than the voltage resolution of the $\mathrm{ADC}$ at all frequencies of interest. In other words, the perturbation amplitude must be greater than single Least Significant Bit (LSB) of the ADC. Otherwise, the signal will be buried in the digital quantization noise and will remain undetected during the frequency response measurements. Based on the above analysis, the following boundary conditions decide the amplitude of the perturbation signal:

a) For frequencies below the crossover frequency, lowperturbation amplitude satisfies a high SNR on the transfer function. However, high gain of the integrator necessitates superimposing a large AC perturbation of the order of $100 \mathrm{mV}$ to measure the small-signal response of the output voltage,

b) For frequencies above the crossover frequency, a high SNR on the duty signal, i.e. a large signal on the duty necessitates superimposing a small amplitude of the perturbation to achieve a given SNR on the transfer function,

c) For intermediate frequencies, the perturbation amplitude must be relatively low to avoid clipping of the output signal.

The multi-tone sinusoid reduces measurement time, as the transients that appear at each frequency sweep will only be present at the first perturbation frequency. The periodic and harmonically-related perturbation signal defined by the amplitude $A_{p}$ and frequency $F_{p}$ describes

$\mathrm{p}(\mathrm{n})=\sum_{\mathrm{k}=1}^{\mathrm{F}} \mathrm{A}_{\mathrm{p}_{\mathrm{k}}} \cdot \sin \left(\frac{2 \pi n}{N} 2^{k}+\theta_{\mathrm{p}_{\mathrm{k}}}\right)$

where $F$ defines number of frequency domain data samples, $\mathrm{N}$ defines the length of the sequence as in power of two and $\theta_{\mathrm{p}}$ is the phase between the different sinusoids. The phase is defined zero for initial measurements.

A similar multi-

\section{B. Non-Parametric System Identification}

It is recognized that frequency-domain identification in continuous-time systems with the BL assumption associates the system model coefficients with the power-stage components. Therefore, frequency domain identification is best suited to perform detection of anomalies in the components and the power converter.

The Fourier transform of a finite-length $N$ of a sequence $x(n)$, i.e.

$$
x(n)=\left\{\begin{array}{c}
0 \text { for } n<0 \\
x(n) \text { for } n \geq N
\end{array}\right.
$$$$
X(\omega)=\sum_{n=0}^{N-1} x(n) e^{-j \omega n}
$$

where $0 \leq \omega \leq 2 \pi$ is the angular frequency and $N$ is the total number of integer samples in a single period. The DFT of a real-valued sequence $x(n)$ sampled at equally spaced frequencies $\omega=2 \pi k /{ }_{N}$ where $k=0,1,2 \ldots N-1$ has a complex spectrum defined by

$$
X(k)=\sum_{n=0}^{N-1} x(n) e^{-j \frac{2 \pi k}{N} n}
$$

Generally, the length of the sequence $N$ is fixed and an integer power of two. The rationale behind this selection is because memory address in the majority of digital controllers is byte-addressable rather than decimal-addressable, which minimizes leakage errors in DFT computation.

The above complex-valued discrete-time sequence can also be represented as the sum of the in-phase and quadrature components, i.e. the $X(k)=X_{I}(k)+j X_{Q}(k)$ where $X_{I}(k)$ and $X_{Q}(k)$ represent real-valued discrete quadrature sequences representing real and imaginary components. These quadrature components are the replicas of the sampled sequence multiplied by the sine and cosine of the perturbation frequency $\omega_{k}=\frac{2 \pi k}{N}$ yields

$X_{I}(k)=\frac{2}{N} \sum_{n=0}^{N-1} x(n) \sin \left(\frac{2 \pi k}{N} n\right)$

$X_{Q}(k)=\frac{2}{N} \sum_{n=0}^{N-1} x(n) \cos \left(\frac{2 \pi k}{N} n\right)$

Similarly, the sampled output sequence gives

$$
\begin{aligned}
& Y_{I}(k)=\frac{2}{N} \sum_{n=0}^{N-1} y(n) \sin \left(\frac{2 \pi k}{N} n\right) \\
& Y_{Q}(k)=\frac{2}{N} \sum_{n=0}^{N-1} y(n) \cos \left(\frac{2 \pi k}{N} n\right)
\end{aligned}
$$

The sampled complex in-phase and quadrature sequence at a specific perturbation frequency finally provides the magnitude and phase response of a sequence at that frequency

$$
\begin{aligned}
& |H(k)|=\sqrt{\frac{\left(Y_{I}(k)\right)^{2}+\left(Y_{Q}(k)\right)^{2}}{\left(X_{I}(k)\right)^{2}+\left(X_{Q}(k)\right)^{2}}} \\
& \arg (H(k))=\tan ^{-1}\left(\frac{Y_{I}(k)+j Y_{Q}(k)}{X_{I}(k)+j X_{Q}(k)}\right) .
\end{aligned}
$$

The Frequency Response Function (FRF) algorithm based on the concept of synchronous IQ demodulation accurately demodulates the quadrature phase signals by multiplying the sampled output voltage and duty signal by sine and cosine signals. The sine and cosine signals are initialized at the beginning of the algorithm. The algorithm stores steady state, quadrature output voltage and duty signals in six separate column vectors of size $A c q \times F$, where the first $F$ rows of a 
column vector contain data of a single acquisition for all the perturbed frequencies and $A c q$ as the number of acquisitions. For example, if the column vector length is $64 \times 10$, the first ten rows correspond to data for 10 perturbation frequencies of a single acquisition. The six column vectors comprise $I V_{\mathrm{o}}(k)$-the in-phase component of the output voltage, $Q V_{\mathrm{o}}(k)$-the quadrature phase component of the output voltage, $I D(k)$-the in-phase component of the duty signal, $Q D(k)$-the quadrature phase component of the duty signal, SumVout - the steady state value of the output voltage and SumDuty - the steady state value of the duty signal. The four quadrature phase sequences are

$$
\begin{aligned}
& I V_{\mathrm{o}}(k)=\frac{2}{N} \sum_{n=0}^{N-1} v_{o}(n) \sin \left(\frac{2 \pi k}{N} n\right) \\
& Q V_{\mathrm{o}}(k)=\frac{2}{N} \sum_{n=0}^{N-1} v_{o}(n) \cos \left(\frac{2 \pi k}{N} n\right) \\
& D(k)=\frac{2}{N} \sum_{n=0}^{N-1} d(n) \sin \left(\frac{2 \pi k}{N} n\right) \\
& Q D(k)=\frac{2}{N} \sum_{n=0}^{N-1} d(n) \cos \left(\frac{2 \pi k}{N} n\right)
\end{aligned}
$$

The data from these four vectors $\left(I V_{\mathrm{o}}(k), Q V_{\mathrm{o}}(k), I D(k)\right.$, and $Q D(k))$ is re-arranged to construct the output voltage matrix $V_{\mathrm{o}_{(A c q \times F)}}$ (16) and duty signal $D_{(A c q \times F)}$ (17) matrix of size $A c q \times F$ where the rows $A c q$ define the number of acquisitions $=64$ and the columns define the number of perturbation frequencies $=10$. The first column represents the start frequency $97.65 \mathrm{~Hz}$ and last column represents the end frequency $50 \mathrm{kHz}$.

$$
\begin{aligned}
& V_{\mathrm{o}_{(A c q \times F)}}=
\end{aligned}
$$

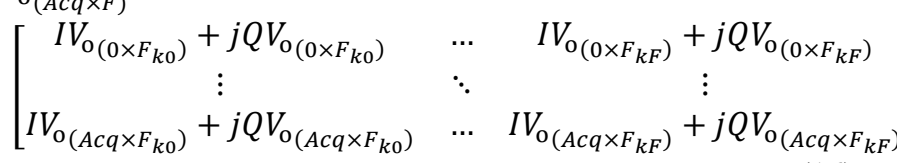

$$
\begin{aligned}
& \begin{array}{l}
D_{(A c q \times F)}= \\
{\left[\begin{array}{ccc}
I D_{\left(0 \times F_{k 0}\right)}+j Q D_{\left(0 \times F_{k 0}\right)} & \ldots & I D_{\left(0 \times F_{k F}\right)}+j Q D_{\left(0 \times F_{k F}\right)} \\
\vdots & \ddots & \vdots \\
I D_{\left(A c q \times F_{k 0}\right)}+j Q D_{\left(A c q \times F_{k 0}\right)} & \ldots & I D_{\left(A c q \times F_{k F}\right)}+j Q D_{\left(A c q \times F_{k F}\right)}
\end{array}\right]}
\end{array}
\end{aligned}
$$

The complex division of $V_{\mathrm{o}_{(A c q \times F)}}$ and $D_{(A c q \times F)}$ matrix then constructs the complex transfer function matrix

$H_{(A c q \times F)}=\left[\begin{array}{ccc}H_{\left(0 \times F_{k 0}\right)} & \ldots & H_{\left(0 \times F_{k F}\right)} \\ \vdots & \ddots & \vdots \\ H_{\left(A c q \times F_{k 0}\right)} & \ldots & H_{\left(A c q \times F_{k F}\right)}\end{array}\right]$

Where $H_{\left(0 \times F_{k 0}\right)}=\frac{I V_{\mathrm{o}\left(0 \times F_{k 0}\right)}+j Q V_{\mathrm{o}\left(0 \times F_{k 0}\right)}}{I D_{\left(0 \times F_{k 0}\right)}+j Q D_{\left(0 \times F_{k 0}\right)}}$

\begin{tabular}{|c|c|}
\hline Step & Equation \\
\hline \multirow{2}{*}{1} & Define numerator and denominator vectors \\
\hline & $D=\left[\begin{array}{lll}d_{0} & d_{1} & d_{2}\end{array}\right]^{T}, N=\left[\begin{array}{ll}n_{0} & n_{1}\end{array}\right]^{T}$ \\
\hline 2 & $\begin{array}{l}\text { Define frequency column vector } \text { freq }_{k}= \\
{\left[\begin{array}{lll}\text { freq }_{0} & \ldots & \text { freq }_{k}\end{array}\right]^{T} \text { where } \mathrm{k}=0,1,2 \ldots \mathrm{F}}\end{array}$ \\
\hline 3 & Initialize $\mathrm{i}=0 \ldots 20, \mathrm{~W}=0, \mathrm{~d}_{0}=1, \mathrm{~d}_{1}, \mathrm{~d}_{2}, \ldots \mathrm{d}_{\mathrm{d}}=0, \mathrm{~N}=0$ \\
\hline 4 & Calculate weighing matrix $W_{i_{k}}=\frac{1}{\left|D_{i_{k}}\right|\left|H_{N P_{k}}\right|}$ \\
\hline \multirow[t]{2}{*}{5} & $\begin{array}{l}\text { Calculatc statc variablc vector } S_{i_{k}}=N_{i_{k}}-D_{i_{k}} H_{N P_{k}} \\
\text { where } N_{i_{k}} \text { and } D_{i_{k}} \text { define the column vector based on the } \\
\text { order of numeratorand denominator vectors }\end{array}$ \\
\hline & $N_{i_{k}}=\left[\begin{array}{ll}s^{0} & s^{1}\end{array}\right]^{T}, D_{i_{k}}=\left[\begin{array}{lll}s^{0} & s^{1} & s^{2}\end{array}\right]^{T}$ \\
\hline 6 & Relative error function is estimated $V_{i_{k}}=S_{i_{k}} W_{i}$ \\
\hline 7 & Calculate $P_{i_{k}}=\sum_{k=0}^{F} \overline{V_{i_{k}}{ }^{T}} \cdot V_{i_{k}}$ \\
\hline 8 & Calculate $Q_{i_{k}}=W_{i} H_{N P_{k}}$ \\
\hline 9 & $\hat{\beta}_{\mathrm{W}_{i}}=\sum_{i}\left[\left(\sum_{k=0}^{F} \overline{V_{i_{k}}{ }^{T}} \cdot V_{i_{k}}\right) \quad\left(\sum_{k=0}^{F} Q_{i_{k}} \overline{V_{i_{k}}{ }^{T}}\right)\right]$ \\
\hline 10 & Go back to step 1 \\
\hline
\end{tabular}

From the above analysis, a non-parametric estimate of the continuous-time system model $H_{N P}\left(\mathrm{~s}_{\mathrm{k}}\right)$ expressed as the ratio
TABLE II

\section{RECURSIVE WEIGHTED LEAST SQUARE ALGORITHM}

of output to input response

$H_{N P}\left(\mathrm{~s}_{\mathrm{k}}\right)=\frac{V_{\mathrm{o}}\left(\mathrm{s}_{\mathrm{k}}\right)}{D\left(\mathrm{~s}_{\mathrm{k}}\right)}=H_{\mathrm{I}}\left(\mathrm{s}_{\mathrm{k}}\right)+\mathrm{j} \mathrm{H} \mathrm{H}_{\mathrm{Q}}\left(\mathrm{s}_{\mathrm{k}}\right)$

calculates the system magnitude and phase response i.e. magnitude and phase matrices for all the perturbation frequencies defined by (20) and (21)

$\left|H_{N P}\left(\mathrm{~s}_{\mathrm{k}}\right)\right|=\sqrt{\left(H_{\mathrm{I}}\left(\mathrm{s}_{\mathrm{k}}\right)\right)^{2}+\left(H_{\mathrm{Q}}\left(\mathrm{s}_{\mathrm{k}}\right)\right)^{2}}$

$\varphi\left(H\left(\mathrm{~s}_{\mathrm{k}}\right)\right)=\tan ^{-1}\left(\frac{H_{\mathrm{Q}}\left(\mathrm{s}_{\mathrm{k}}\right)}{H_{\mathrm{I}}\left(\mathrm{s}_{\mathrm{k}}\right)}\right)$

where $s_{k}=j \omega_{k}$ defines the Laplace transform variable evaluated along the imaginary axis at DFT frequency $\mathrm{k}$.

From these FRF measurements at different perturbation frequencies, a non-parametric system model is estimated prior to parametric identification of the model. This identification enables understanding the source of different uncertainties and nonlinear distortions in the system and ultimately, extracting a non-parametric noise model.

\section{Parametric Identification of DC-DC converter}

The process of parametric model identification is dependent on the non-parametric FRF measurements. The uncertainties in the measured data, such as quantization noise, switching noise, measurement errors, and errors including DFT noise, etc., influence accurate identification of the system model. These uncertainties further lead to inaccurate estimation of model parameters, extraction of component values, and erroneous 
interpretation of degradation. Therefore, it is emphasized throughout the development of the health monitoring method that uncertainties introduced by the digital control and the measurement process must be minimized. In this study, the majority of these uncertainties are eliminated by using a periodic excitation and synchronous demodulation of system dynamical characteristics.

For parametric identification, the model estimator targets reducing the error between the measured transfer function $\mathrm{H}_{\mathrm{NP}}\left(\mathrm{s}_{\mathrm{k}}\right)$ and the assumed system model $\mathrm{H}_{\mathrm{P}}\left(\mathrm{s}_{\mathrm{k}}\right)$. The method assumes the ratio of frequency-dependent complex polynomials $N\left(\mathrm{~s}_{\mathrm{k}}\right)$ and $D\left(\mathrm{~s}_{\mathrm{k}}\right)$, i.e. Laplace rational fraction in continuoustime domain as the system model.

The identification process states the system model as a solution to the normal equation of the form

$$
\begin{aligned}
& \hat{\beta}_{\mathrm{W}_{i}}= \\
& \sum_{i}\left[\left(\sum_{k=0}^{F} \overline{\left(S_{l_{k}} W_{l}\right)^{T}} \cdot\left(S_{i_{k}} W_{i}\right)\right)^{-1}\left(\sum_{k=0}^{F}\left(W_{i} H_{N P_{k}}\right) \cdot \overline{\left(S_{l_{k}} W_{l}\right)^{T}}\right)\right]
\end{aligned}
$$

where $\hat{\beta}_{\mathrm{W}}$ represents the best model fit of the model coefficients, $W$ states the real-value diagonal weighing matrix. The individual weights of the weighing matrix are strictly positive $w_{i j} \geq 0$. $S$ represents the state variable vector containing bias function and coefficients as its elements. The '.' denotes the dot product, ${ }^{-}$denotes the complex conjugate, and $T$ denotes the transpose. $H_{N P}$ is a column vector containing non-parametric FRF measurements.

The parametric model identification algorithm based Recursive Weighted Least Square (WLS) is executed in MathCAD. In steps 1 and 2 , the algorithm defines and initializes the denominator, the numerator, and the frequency vectors. The elements of the weighing matrix, the numerator and denominator vectors are initialized to zero except $d_{0}$ is constrained to 1.0 in the next step. The WLS estimator takes the non-parametric FRF measurement column vector and denominator vector as the input to evaluate the weighing matrix, i.e. for $i=0$, the unknown weighing function is defined based on prior analysis of the measured transfer function in step 4. Consequently, the algorithm estimates the state variable vector and the cost function based on the selected order of the numerator and the denominator vectors in step 5. The next step entails estimating the relative error function. This modifies to matrix $P$ to obtain the first part of the said normal equation. In step 8 , the second part of the normal equation is approximated by weighing the individual experimental data points. Finally, the best estimate of the system model is evaluated in step 9. The entire process recursively minimizes the relative error instead of the absolute error on the model estimates. This algorithm is limited to only one iteration. However, the number of iterations can be increased for an optimum result. This relative error criterion combined with a weighing and iterative process yields best fit for the system model. The estimated best-fit model defined in the form of a Laplace rational fraction enables extracting the model coefficients and the component values thereafter.

\section{Parameter Extraction}

The majority of the research on system identification of power converters identify non-parametric system response. However, they fail to estimate the model (numerator and denominator) coefficients and the subsequent power-stage component values that give a clear indication of the deviation of the circuit components from their actual values. The identification of model parameters and subsequent circuit components is essential as it detects drift in the system and the components from its healthy state and enables accurate detection of degradation.

Reproducing the results, the identified model defines an norder system depending on the power-stage network. Using the equivalent Laplace model of a power-stage network defined by

$H\left(s_{k}\right)=\frac{n_{0}+s_{k} n_{1}+s_{k}^{2} n_{2}+. .}{d_{0}+s_{k} d_{1}+s_{k}^{2} d_{2}+.}$

where the model coefficients $n_{0}, n_{1}, d_{0}, d_{1}$ and $d_{2}$ represent the power-stage network elements.

For a basic DC-DC buck converter, the power-stage network (Fig.1) forms a second-order system as it contains two energy storing passive components. Therefore, the equivalent Laplace model of a second-order is defined by

$H\left(s_{k}\right)=\frac{n_{0}+s_{k} n_{1}}{d_{0}+s_{k} d_{1}+s_{k}^{2} d_{2}}$

where the model coefficients can be evaluated from the transfer function equivalent to

$\mathrm{H}(\mathrm{s})=\frac{v_{o}(s)}{d(s)}=\mathrm{K}\left(\frac{\mathrm{Z}_{\text {out }}}{\mathrm{Z}_{\text {out }}+\mathrm{Z}}\right)$

where

$\mathrm{K}=v_{\text {in }}+v_{S 2 \text { (Knee) }}$

$\mathrm{Z}_{\text {out }}=\frac{\mathrm{R}_{\text {Load }} \times\left(r_{C}+\frac{1}{s C}\right)}{\mathrm{R}_{\text {Load }}+\left(r_{C}+\frac{1}{s C}\right)}$

and

$Z=s L+r_{L+S}$

where the combined resistance of $r_{L}, r_{S 1}, r_{S 2}$ and the shunt resistance $r_{\text {Shunt }}$ is defined by

$\mathrm{r}_{\mathrm{L}+\mathrm{S}}=\mathrm{r}_{\mathrm{L}}+\mathrm{D} \mathrm{r}_{\mathrm{S} 1}+(1-\mathrm{D}) \mathrm{r}_{\mathrm{S} 2}+\mathrm{r}_{\text {Shunt }}$

and $v_{S 2 \text { (Knee) }}$ defines the knee voltage of the switch S2.

Substituting (26) and (27) in (25) evaluates second-order transfer function to

$$
\begin{aligned}
& H(s)=K \frac{\frac{R_{L o a d}}{R_{L o a d}+r_{L+S}}+s\left(\frac{R_{L o a d} r_{C} C}{R_{L o a d}+r_{L+S}}\right)}{1+} \frac{s\left(\frac{\left(C\left(R_{L o a d} r_{C}+r_{L+S} R_{L o a d}+r_{L+S} r_{C}\right)+L\right)}{R_{L o a d}+r_{L}+S}\right)+}{s} \\
& \mathrm{~s}^{2}\left(\frac{\mathrm{LC}\left(\mathrm{R}_{\mathrm{Load}}+\mathrm{r}_{\mathrm{C}}\right)}{\mathrm{R}_{\mathrm{Load}}+\mathrm{r}_{\mathrm{L}+\mathrm{S}}}\right)
\end{aligned}
$$


Therefore,

$$
\begin{aligned}
& \mathrm{n}_{0}=\mathrm{K} \frac{\mathrm{R}_{\mathrm{Load}}}{\left(\mathrm{R}_{\mathrm{Load}}+\mathrm{r}_{\mathrm{L}+\mathrm{S}}\right)} \\
& \mathrm{n}_{1}=\mathrm{K} \frac{\mathrm{R}_{\mathrm{Load}}}{\left(\mathrm{R}_{\mathrm{Load}}+\mathrm{r}_{\mathrm{L}+\mathrm{S}}\right)} \mathrm{Cr} \mathrm{r}_{\mathrm{c}} \\
& \mathrm{d}_{0}=1 \\
& \mathrm{~d}_{1}=\frac{\mathrm{r}_{\mathrm{L}+\mathrm{S}} \mathrm{C}\left(\mathrm{R}_{\mathrm{Load}}+\mathrm{r}_{\mathrm{C}}\right)+\mathrm{CR}_{\mathrm{Load}} \mathrm{r}_{\mathrm{C}}+\mathrm{L}}{\left(\mathrm{R}_{\mathrm{Load}}+\mathrm{r}_{\mathrm{L}+\mathrm{S}}\right)} \\
& \mathrm{d}_{2}=\mathrm{LC} \frac{\left(\mathrm{R}_{\mathrm{Load}}+\mathrm{r}_{\mathrm{C}}\right)}{\left(\mathrm{R}_{\mathrm{Load}}+\mathrm{r}_{\mathrm{L}+\mathrm{S}}\right)}
\end{aligned}
$$

\section{EXPERIMENTAL INVESTIGATION}

Fig. 1. provides a block diagram of a synchronous POL converter which is powered from input $v_{i n}(t)=3.3 \mathrm{~V}$ to provide a regulated low-voltage output $v_{o}(t)=1.2 \mathrm{~V}$. The power-stage circuit component inductor $\mathrm{L}$, output filter capacitor $\mathrm{C}$ and its ESR $r_{C}$, resistance of the switching devices, and the output load $R_{\text {Load }}$ are defined as $L=150 \mu \mathrm{H}, C=220 \mu \mathrm{F}, r_{C}=90 \mathrm{~m} \Omega$ (measured at $8 \mathrm{kHz}$ ), $r_{L+S}=840 \mathrm{~m} \Omega$ and $R_{\text {Load }}=10 \Omega$. $S 1$ and $S 2$ are the synchronous switching devices. The switching frequency of the converter is $200 \mathrm{kHz}$.

The rationale for using a low-voltage converter is to emulate the power supply rail of the digital load, such as FPGA constrained by the tight voltage tolerance. The typical tolerance requirement of the low-voltage power rail is $2-3 \%$, compared to $5-6 \%$ of a high-voltage power rail (for instance, $15 \mathrm{~V}$ ). This restriction on the selection of the low-voltage power converter enables recognizing the implementation of the method on lowvoltage industrial applications.

A DSP-based microcontroller including 32-bit floating-point arithmetic and an integrated ADC, PWM, and an embedded Random Access Memory (RAM), is used to regulate the control loop and act as an embedded sensor for data acquisition. The digital controller Piccolo ${ }^{\mathrm{TM}}$ F2806x from Texas Instruments (TI) interfaces to the POL converter via 16-pin connector and $\mathrm{PC}$ via an on-board USB as shown in Fig. 3. The Code Composer Studio (CCS) development tool from TI provides on-board JTAG emulation, access to peripherals such as ADC and PWM, and real-time debug to the control algorithm [48]. The CCS Integrated Development Environment (IDE) controls and monitors the power converter wholly via software reprogrammability and flexibility.

The 12-bit Successive Approximation Register (SAR) ADC

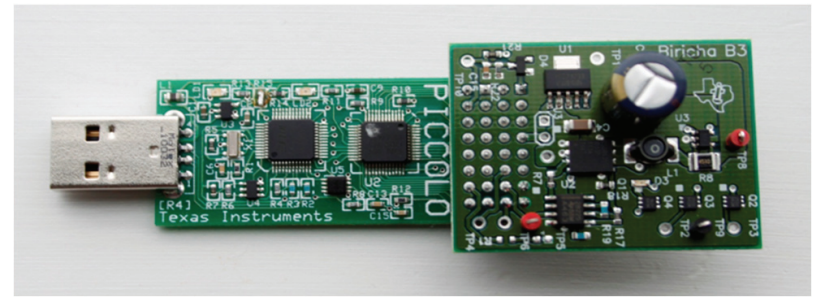

Fig. 3. Experimental Test Module
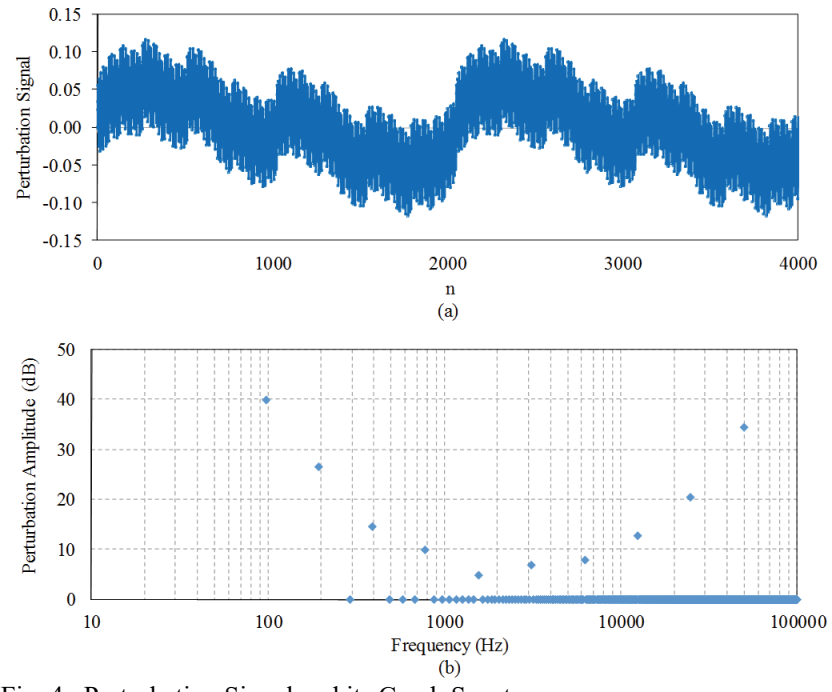

Fig. 4. Perturbation Signal and its Comb Spectrum

with an integrated Sample and Hold $(\mathrm{S} / \mathrm{H})$ circuit samples the output voltage $v_{o}(t)$ at a sampling frequency $F_{S P}$. The timerbased PWM module generates a pulse width modulated duty signal $d(t)$ after the addition of an excitation signal to the compensated control voltage. The voltage resolution of the timer module, defined as the ratio of the system clock period and the user-defined switching period $T_{S}$, governs the resolution of the duty signal. The Piccolo PWM timer module provides a duty signal resolution of $2.5 \mathrm{mV}$, given the system period is $12.5 \mathrm{~ns}$ and a switching period is $5 \mu \mathrm{s}$ [49]. To avoid limit cycling [50], Micro Edge Positioning (MEP) integrated in the controller provides a much finer PWM resolution of the order of $36 \mu \mathrm{V}$. The CCS Integrated Development Environment (IDE) provides the selection of the reference signal for the integrated ADC, the design of discrete 3pole-3zero digital IIR filter, and the configuration of the digital pulse-width modulator. It is necessary to acknowledge that the input filter modifies the control-to-output system response. However, for initial assessment of the proposed methodology, the study does not consider its design and implications.

The design of the low-voltage AC perturbation is such that the maximum variation on the output voltage is within $\pm 1.0 \%$ of the nominal value and the maximum variation on duty is $\pm 3.0 \%- \pm 5.0 \%$ of the nominal to achieve a constant SNR across the desired frequency spectrum. Fig. 4(a) illustrates the outline of the digital perturbation written in $\mathrm{C}++$ and programmed in the digital controller. The amplitude of the perturbation is greater than the voltage resolution of the ADC, ensuring the signal will not be buried in the quantization noise and the analogue noise of the system.

Since the perturbation signal is the sum of harmonically related sinusoids, the frequency spectrum of the signal represents a comb spectrum as shown in Fig. 4(b). The discrete tones are synchronized with the switching frequency of the digital controller to largely minimize leakage errors and the effect of harmonics. Moreover, the factor of two between each frequency gives a programming advantage where the variable 'frequency' is right shifted every time to obtain the next 

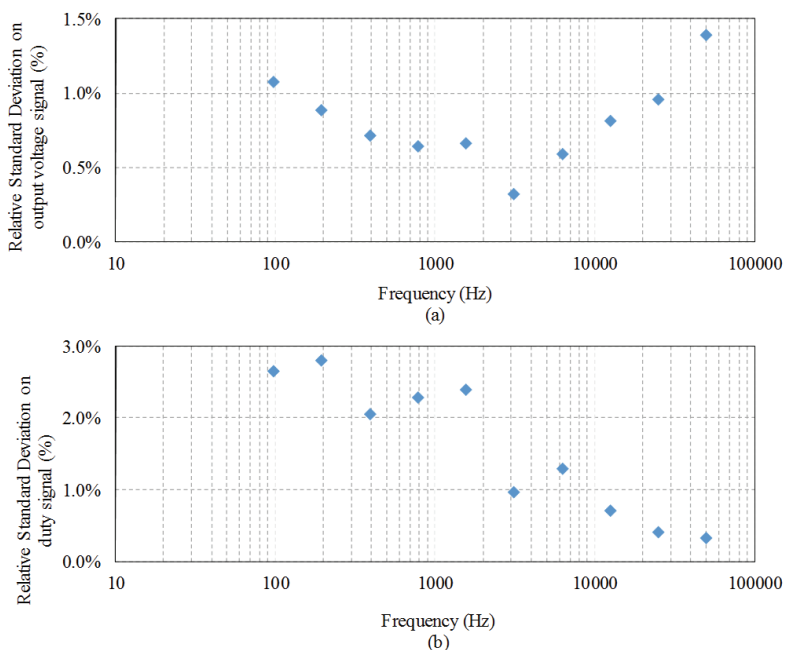

Fig. 5. Relative Standard Deviation on output voltage and duty signal

frequency thereby saving a lot of computational time. The comb spectrum only injects ten frequency sweeps to obtain the frequency response of the entire system.

The relative standard deviation on the output voltage and the duty signals in Fig. 5(a) and (b) reveal that the injected multitone perturbation limits the duty signal within $3 \%$ of its nominal value and the output voltage signal within $2 \%$, i.e. $24 \mathrm{mV}$ for a $1.2 \mathrm{~V}$ signal, for all the frequencies of interest. Superimposing an optimized multi-tone signal at frequencies of interest does not disturb the steady state response of the converter. There is low noise on the output voltage and the duty signal, considering there are other uncertainties in the system, such as quantization noise of the samplers, measurement noise, and the inherent analogue noise of the system. Consequently, this low percentage of the relative standard deviation on the input (output voltage) and the output (duty) indicates low variability on the data set, and hence a high SNR on the transfer function.

The uncertainty on the magnitude and phase response describes how the SNR is preserved across the defined
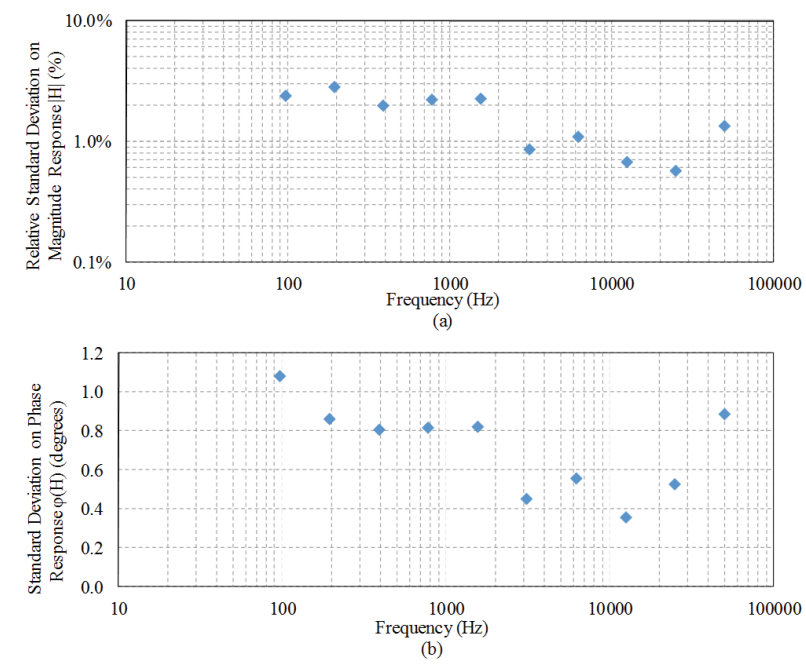

Fig. 6. Relative Standard Deviation on $|\mathrm{H}(\mathrm{s})|$ and $\varphi(\mathrm{H}(\mathrm{s}))$

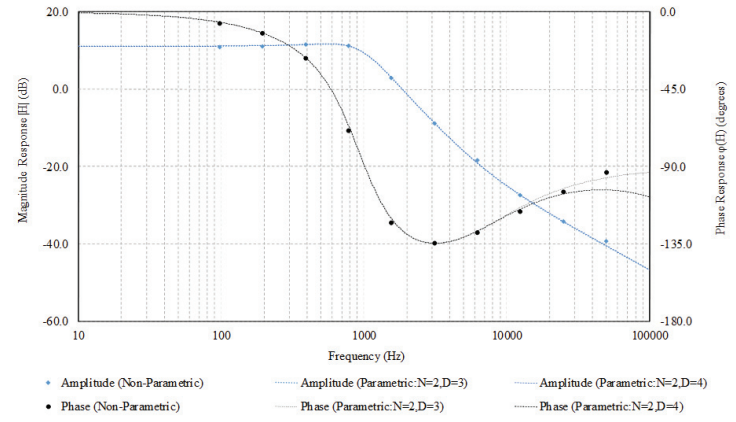

Fig. 7. Non-Parametric Response vs Parametric System Model

frequencies of interest as depicted in Fig. 6(a) and (b). The high variability at low frequencies, compared to higher frequencies on both magnitude and phase indicate more noise at the lower frequencies. This noise can be the quantization noise of the ADC, DPWM or the analogue noise of the system. However, the origin of this variability on the response is not known.

The non-parametric system model in Fig. 7 depict that the amplitude response asymptotically decays by $-20 \mathrm{~dB} /$ decade at frequency $1 \mathrm{kHz}$ and further rolls off by $-20 \mathrm{~dB} /$ decade at 5 $\mathrm{kHz}$. This graphical interpretation allows assuming a secondorder parametric system model with numerator and denominator polynomial of the order of two and three respectively. The close match between the non-parametric response of the system and the assumed parametric secondorder model of the system in Fig. 8 indicates that minimizing relative error using a Recursive WLS estimator provides a good fit of the model over a wide frequency range from $10 \mathrm{~Hz}$ to $10 \mathrm{kHz}$. However, the model is verified against a third-order system with $\mathrm{N}=2$ and $\mathrm{D}=4$. The response evidently signifies the difference in phase response with a second and a third-order system at the higher frequencies.

For parameter extraction of power-stage component values, the inductance $L$ and the output load $R_{\text {Load }}$ are assumed to be $150 \mu \mathrm{H}$ and $10 \Omega$, similar to the experimental test module and as assumed in [12]. Inductors are reliable than capacitors, however, they show large tolerance in their values. The method presented herein does not take into account these tolerances for parameter extraction. The actual component values on experimental test module are measured using an RLC meter. Using (31)-(35), the capacitance, ESR of the output filter capacitor, and the resistance of the inductor including the switches are extracted. The extracted capacitance value is within the $20 \%$ tolerance of the actual value as shown in Table III. Similarly, the extracted ESR shows a consistent result with the actual ESR on the board. It is important to mention that the equivalent Laplace model considers a simple model of the capacitor against a realistic RC ladder model of the electrolytic capacitor.

The parametric identification of the system model with relative minimization of the cost function across the logarithmic frequency range provides accurate extraction of the powerstage component values of the power converter, compared to 

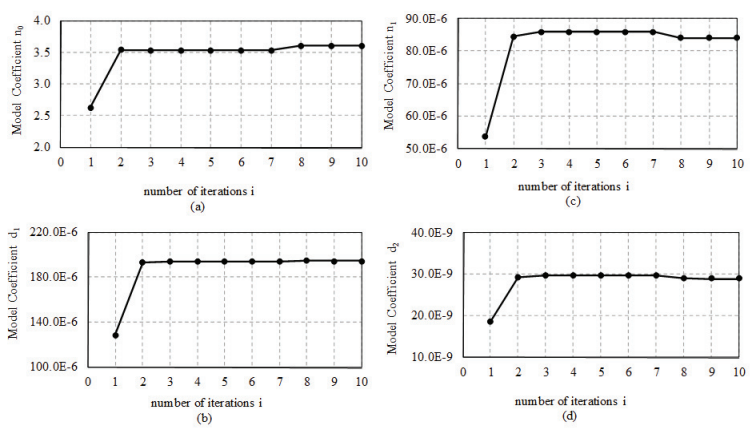

Fig. 8. Convergence of System Model Coefficient with number of iterations

the actual component values on the buck converter. The above results signify that the proposed parametric identification can be used to detect degradations in switch-mode power supply topologies whilst proper care is taken for the design of perturbation selection, non-parametric FRF measurements, and extraction of model coefficients to minimize the variance on the transfer function.

An important result of the model coefficients in Fig. 8 demonstrates that all model coefficients converge within one or a maximum of two iterations with an accuracy of $\pm 1 \%$. This reduces the complexity of the model and provides fast identification of the system.

\section{Aging Detection CApability For Switch-Mode CONVERTERS}

The majority of the health-monitoring research community performs different acceleration tests such as HAST or apply environmental or operational stress to the system to predict the end of life of the system and/or its components. Subsequently, the continuous monitoring and measurement of certain system parameters along with their theoretical models provide knowledge of anomalies in the system and the components. These predictions are formulated when the system is functioning outside its normal operating conditions. The knowledge of degradation of a system and its components functioning within its operating conditions is not available. By contrast, this study proposes detecting anomalies in passive components by operating the system under normal working conditions.

One method could be to increase the circuit component value and recognize that the health-monitoring methodology detects the system change. For example, an external capacitor connected in parallel with the output capacitor would increase the overall capacitance of the output filter. It follows from here that if the health-monitoring technique detects the increased external capacitance, it can be argued that the methodology can be used to detect anomalies or aging degradations in passive electronic components.

The above hypothesis is experimentally validated by implementing the same methodology on the modified circuit illustrated in Fig. 9 without changing the design and implementation of the $\mathrm{ADC}$, digital compensator, and the

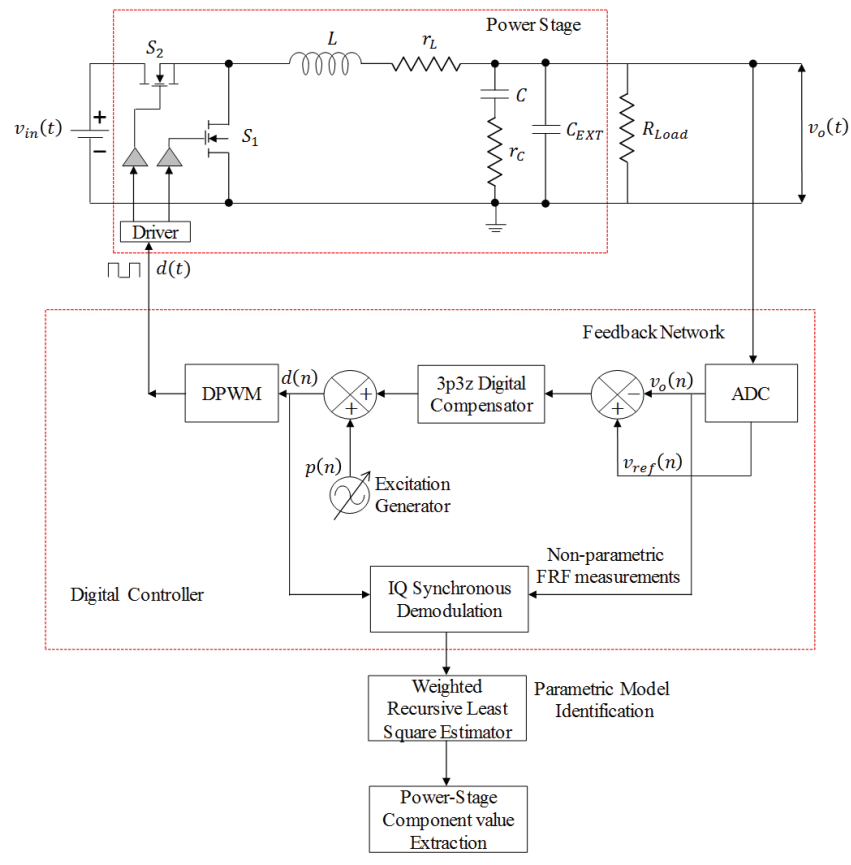

Fig. 9. Modified Block Diagram of Parametric Identification Based HealthMonitoring on Digitally-Controlled DC-DC Buck Converter for Aging Detection on Power-Stage Network Elements

DPWM. The measurement algorithm is encoded in the Piccolo $^{\mathrm{TM}}$ F2806x while system identification and the parameter extraction algorithm is implemented in MathCAD.

The modified power-stage depicts the addition of external capacitance $C_{E X T}$ in parallel to the output capacitor. Twelve ceramic capacitors with values ranging from $2 \mu \mathrm{F}$ to $30 \mu \mathrm{F}$ are added sequentially as external capacitance in the circuit. The tolerance of selected ceramic capacitors is low, $1 \%$, compared to $20 \%$ tolerance of the output electrolytic capacitor. In addition, the ESR of ceramic capacitors is trivial in comparison to the ESR of the electrolytic output capacitor. The selection of the external capacitor assures that the transfer function will be stable and within its operating conditions.

The non-parametric frequency response measurements by perturbing the modified system followed by parametric system identification and parameter extraction using WLS, extracts Laplace rational fraction coefficients and the associated powerstage component values. The rational fraction coefficients and the value of total capacitance (with $C_{E X T}$ ) is extracted assuming inductance $L$ and the output load $R_{\text {Load }}$ are $150 \mu \mathrm{H}$ and $10 \Omega$, similar to the experimental test module.

The results describe that the overall capacitance on the modified experimental test module follows a rising trend

TABLE III

COMPARISON OF ACTUAL AND EXTRACTED POWER-STAGE COMPONENT VALUES

\begin{tabular}{ccc}
\hline Power Stage Component & $\begin{array}{c}\text { Actual Component Value on } \\
\text { Experimental Test Module }\end{array}$ & $\begin{array}{c}\text { Extracted Component Value } \\
\text { from the Proposed Algorithm }\end{array}$ \\
\hline $\mathrm{C}(\mu \mathrm{F})$ & 220.0 & 217.8 \\
\hline $\begin{array}{c}\mathrm{r}_{\mathrm{C}}(\mathrm{m} \Omega) \\
(\text { measured at } 8 \mathrm{kHz})\end{array}$ & 90.0 & 108.0 \\
\end{tabular}




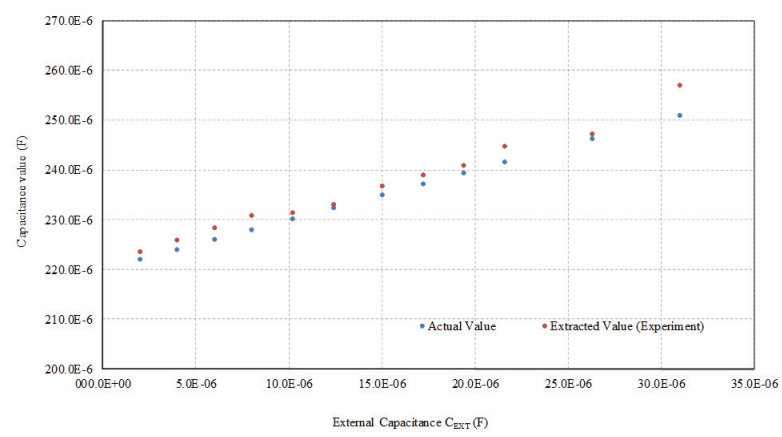

Fig. 10. Comparison between Actual Capacitance on the Modified Circuit and Extracted Value from Parametric Identification Algorithm

indicating an increase in overall capacitance of the circuit as shown in Fig. 10. Evidently, the extraction algorithm accurately estimates the overall capacitance across the output of a buck converter, taking into consideration the tolerance of the components and the uncertainty associated with the measurements, modelling, and component extraction.

\section{CONCLUSION}

In this paper, aging detection capability for a switch-mode power converter is developed. Prior to detecting aging degradations, a parametric system identification based health monitoring methodology is developed and presented. The health monitoring technique involved i) non-parametric identification of the system by injecting small multi-tone sinusoidal perturbation (of the order of few $\mathrm{mVs}$ ) at only ten frequencies within the closed loop; ii) Parametric identification of the system using a Recursive Weighted Least Square estimator; and iii) parameter extraction of power-stage component values assuming a Laplace rational function of the system. The effectiveness of the health monitoring technique is observed by accurate estimation of the parametric model and extraction of the component values. Finally, the aging detection capability of the proposed technique is established by inserting additional capacitance across the output. The detection procedure involves the use of different capacitance values to recognize its effect on the overall capacitance of the circuit. The overall capacitance illustrates an increasing trend with increased external capacitance demonstrating the implementation of an accurate detection methodology and demonstrating its usefulness for predicting end-of-life of power-supply components. This methodology is tested and validated on a basic POL converter but can be generalized to other power converter topologies.

\section{REFERENCES}

[1] EPRI (2005, May) Evaluating the Effects of Aging on Electronic Instrument and Control Circuit Boards and Components in Nuclear Power Plants, 1011709, U.S. Department of Energy, Palo Alto, CA, and U.S. Department of Energy.

[2] J.W. Bennett, G.J. Atkinson, B.C. Mecrow, D.J. Atkinson, "FaultTolerant Design Considerations and Control Strategies for Aerospace Drives," IEEE Trans. Ind. Electron., vol. 59, no. 5, pp. 2049-2058, May 2012.
[3] A.S. Sarathi Vasan, B. Long, M. Pecht, "Diagnostics and Prognostics Method for Analog Electronic Circuits," IEEE Trans. Ind. Electron., vol. 60, no. 11, pp. 5277-5291, June 2013.

[4] Y. Xiong, X. Cheng, Z. J. Shen, C. Mi, H. Wu and V. K. Garg "Prognostic and warning system for power-electronic modules in electric, hybrid electric, and fuel-cell vehicles," IEEE Trans. Ind. Electron., vol. 55, no. 6, pp.2268 -2276, May 2008

[5] I. K. Jennions, "Integrated Vehicle Health Management: Perspectives on an Emerging Field," SAE International, 1st ed., Pennsylvania, USA, 2011.

[6] R. Orsagh, D. Brown, M. Roemer, T. Dabnev, and A. Hess, "Prognostic health management for avionics system power supplies," Proc. IEEE Aerosp. Conf., Big Sky, MT, 2005, pp. 3585-3591.

[7] ITRS (2007) International Technology Roadmap for Semiconductors Executive Summary, Overall Roadmap Technology Characteristics, ITRS.

[8] ITRS (2011) International Technology Roadmap for Semiconductors: Executive Summary, Power Supply and Power Dissipation, ITRS.

[9] Y.-M. Chen, H.-C. Wu, M.-W. Chou and K.-Y. Lee, "Online failure prediction of electrolytic capacitors for LC filter of switching-mode power converters", IEEE Trans. Ind. Electron., vol. 55, no. 1, pp.400 406, Jan. 2008.

[10] S. Yang, D. Xiang, A. Bryant, P. Mawby, L. Ran, and P. Tavner, "Condition monitoring for device reliability in power electronic converters - A Review," IEEE Trans. Power Electron., vol. 25, no. 11, pp. $2734-2752$, Nov. 2010.

[11] C. Kulkarni, G. Biswas, X. Koutsoukos, J. Celaya, and K. Goebel, "Integrated diagnostic/prognostic experimental setup for capacitor degradation and health monitoring," Proc. IEEE AUTOTESTCON, Orlando, FL, 2010, pp. 1-7.

[12] A.M.R. Amaral and A.J.M. Cardoso, "On-line fault detection of aluminium electrolytic capacitors, in stepdown DC-DC converters, using input current and output voltage ripple," IET Power Electron., vol.5, no.3, pp.315-322, March 2012.

[13] A. Lahyani, P. Venet, G. Grellet, P. Viverge, "Failure prediction of electrolytic capacitors during operating of a switch mode power supply, " IEEE Trans. Power Electron., vol.13, no.6, pp.1199-1207, Nov. 1998.

[14] A.M.R. Amaral and A.J.M. Cardoso, "An economic technique for estimating the equivalent circuit of aluminum electrolytic capacitors, " IEEE Trans. Instrum. Meas., vol.57, no.12, pp. 2697-2710, Dec. 2008.

[15] A.M.R. Amaral and A.J.M. Cardoso, "A simple offline technique for evaluating the condition of aluminum-electrolytic-capacitors, " IEEE Trans. Ind. Electron., vol.56, no.8, pp. 3230-3237, Aug. 2009.

[16] A.M.R. Amaral and A.J.M. Cardoso, "Simple experimental techniques to characterize capacitors in a wide range of frequencies and temperatures, " IEEE Trans. Instrum. Meas., vol.59, no.5, pp. 1258-1267, May 2010.

[17] Y. Chen, H. Wu, M. Chou, and K. Lee, "Online failure prediction of electrolytic capacitor for LC filter of switching-mode power converters, " IEEE Trans. Ind. Electron., vol. 55, no. 1, pp. 400-406, Jan. 2008.

[18] R. Erickson, and D. Maksimović, "Fundamentals of Power Electronics," 2nd ed., Kluwer, Norwell, MA, 2001.

[19] L. Ljung, "System identification: Theory for the User," 2nd ed., NJ: Prentice-Hall, 1999.

[20] J. Schoukens, R. Pintelon, E. van der Ouderaa, and J. Renneboog, "Survey of excitation signals for FFT based signal analyzers," IEEE Trans. Instrum. Meas., vol. 37, no. 3, pp. 342-352, Sept. 1988.

[21] T. Roinila, M. Vilkko and T. Suntio, "Frequency-response measurement of switched-mode power supplies in the presence of nonlinear distortions," IEEE Trans. Power Electron., vol.25, no.8, pp. 2179-2187, Aug. 2010

[22] M. Shirazi, J. Morroni, A. Dolgov, and D. Maksimovic, "Integration of frequency response measurement capabilities in digital controllers for DC-DC converters," IEEE Trans. Power Electron., vol. 23, no. 5, pp. 2524-2535, Sept. 2008.

[23] B. Miao, R. Zane, and D. Maksimovic, "System identification of power converters with digital control through cross-correlation methods," IEEE Trans. Power Electron., vol. 20, no. 5, pp. 1093-1099, Sept. 2005.

[24] A. Barkley and E. Santi, "Improved online identification of a DC-DC converter and its control loop gain using cross-correlation methods," IEEE Trans. Power Electron., vol.24, no.8, pp. 2021-2031, Aug. 2009.

[25] R. Pintelon, and J. Schoukens, "System Identification: A Frequency Domain Approach," 2nd ed., John Wiley \& Sons, Inc., NJ, 2001.

[26] F. Gonzalez-Espi, E. Figueres, G. Garcera, R. Gonzalez-Medina, and M. Pascual, "Measurement of the loop gain frequency response of digitally 
controlled power converters," IEEE Trans. Ind. Electron., vol. 57, no. 8 , pp. 2785-2796, Nov. 2009.

27] O. Jimenez, O. Lucia, I. Urriza, L.A. Barragan, D. Navarro, "Analysis and Implementation of FPGA-Based Online Parametric Identification Algorithms for Resonant Power Converters," IEEE Trans. Ind. Informat., vol.10, no.2, pp.1144-1153, May 2014

[28] B. Johansson and M. Lenells, "Possibilities of obtaining small-signal models of dc-dc power converters by means of system identification," Proc. Telecommun. Energy Conf., 2000, pp. 65-75.

[29] M. Algreer, M. Armstrong, and D. Giaouris, "Active online system identification of switch mode DC-DC power converter based on efficient recursive DCD-IIR adaptive filter," IEEE Trans. Power Electron., vol. 27, no. 11 , pp. 4425-4435, Mar. 2012.

[30] J. Schoukens, R. Pintelon, and H. Van. Hamme, "Identification of linear dynamic systems using piecewise constant excitations: use, misuse and alternatives," Automatica, vol. 30, no. 7, pp. 1153-1169, July 1994.

[31] B. Miao, R. Zane, and D. Maksimovic, "System identification of power converters with digital control through cross-correlation methods," IEEE Trans. Power Electron., vol. 20, no. 5, pp. 1093-1099, Sept. 2005.

[32] T. Roinila, T. Helin, M. Vilkko, T. Suntio, and H. Koivisto, "Circular correlation based identification of switching power converter with uncertainty analysis using fuzzy density approach," Simul. Model. Pract.Th., vol. 17, no. 6, pp. 1043-1058, Mar. 2009

[33] Y. Zhang, H. Zhou, S. J. Qin, and T. Chai, "Decentralized fault diagnosis of large-scale processes using multiblock kernel partial least squares," IEEE Trans. Ind. Informat., vol. 6, no. 1, pp. 3-10, Feb. 2010.

[34] D. Wang, J. Liu, and R. Srinivasan, "Data-driven soft sensor approach for quality prediction in a refining process," IEEE Trans. Ind. Informat., vol. 6 , no. 1, pp. 11-17, Feb. 2010.

[35] R. Muradore and P. Fiorini, "A PLS-based statistical approach for fault detection and isolation of robotic manipulators," IEEE Trans. Ind. Electron., vol. 59, no. 8, pp. 3167-3175, Aug. 2012.

[36] S. Yin, X. Zhu, O. Kaynak, "Improved PLS Focused on KeyPerformance-Indicator-Related Fault Diagnosis," IEEE Trans. Ind. Electron., vol. 62, no. 3, pp. 1651-1658, Mar. 2015.

[37] X. Zhao, Y. Xue, and T. Wang, "Fault detection of batch process based on multi-way Kernel T-PLS," J. Chem. Pharm. Res., vol. 6, no. 7, pp. 338-346, Jul. 2014.

[38] D. Zhou, G. Li, and S. J. Qin, "Total projection to latent structures for process monitoring," AIChE J., vol. 56, no. 1, pp. 168-178, Jan. 2010

[39] V. Choqueuse, M. E. H. Benbouzid, Y. Amirat, and S. Turri, "Diagnosis of three-phase electrical machines using multidimensional demodulation techniques," IEEE Trans. Ind. Electron., vol. 59, no. 4, pp. 2014-2023, Apr. 2012.

40] C-M. Vong, P-K.Wong, and W- F. Ip, “A new framework of simultaneous fault diagnosis using pairwise probabilistic multi-label classification for time-dependent patterns," IEEE Trans. Ind. Electron., vol. 60, no. 8, pp 3372-3385, Aug. 2013.

[41] J. Gertler and J. Cao, "PCA-based fault diagnosis in the presence of control and dynamics," AIChE J., vol. 50, no. 2, pp. 388-402, Feb. 2004

[42] D. Tsai, S. Wu, and W. Chiu, "Defect detection in solar modules using ICA basis images," IEEE Trans. Ind. Informat., vol. 9, no. 1, pp. 122 131, Feb. 2013

[43] F. Zidani, D. Diallo, M. Benbouzid, and R. Nait-Said, "A fuzzy-based approach for the diagnosis of fault modes in a voltage-fed PWM inverter induction motor drive," IEEE Trans. Ind. Electron., vol. 55, no. 2, pp. 586-593, Feb. 2008

[44] S. Yin, X. Gao, H. Karimi, and X. Zhu, "Study on support vector machine based fault detection in Tennessee Eastman Process," Abstract Appl. Anal., vol. 2014, 2014, Art. ID. 836895.

[45] F. Ye, Z. Zhang, K. Chakrabarty, and X. Gu, "Board-level functional fault diagnosis using multikernel support vector machines and incremental learning," IEEE Trans. Comput.-Aided Design Integr. Circuits Syst., vol. 33, no. 2, pp. 279-290, Feb. 2014

[46] S. Huang, K. K. Tan, and T. H. Lee, "Fault diagnosis and fault-tolerant control in linear drives using the Kalman filter," IEEE Trans. Ind. Electron., vol. 59, no. 11, pp. 4285-4292, Nov. 2012.

[47] P. Lall, R. Lowe, and K. Goebel, "Prognostics health management of electronic systems under mechanical shock and vibration using Kalman filter models and metrics," IEEE Trans. Ind. Electron., vol. 59, no. 11, pp. 4301-4314, Nov. 2012.

[48] Texas Instruments (2014) Code Composer Studio (CCS) Integrated Development Environment (IDE).

[49] TMS320x2806x Piccolo Technical Reference Manual - SPRUH18E, Texas Instruments Incorporated, Texas, 2014
[50] A.V. Peterchev and S. R. Sanders, "Quantization resolution and limit cycling in digitally controlled PWM converters," IEEE Trans. Power Electron., vol.18, no.1, pp.301-308, Jan. 2003.

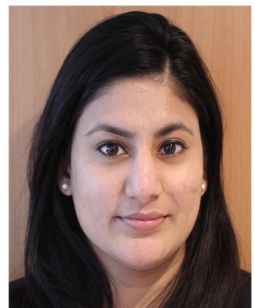

Jaspreet Kaur Mann (M'14) received BEng in Electronics and Communication Engineering from Chitkara University, India in 2007 and MSc degree in Electronics and Computer Engineering from University of Birmingham, United Kingdom, in 2008. From 2008 to 2010, she has worked for Ericsson Limited as a Hardware Design Engineer. In 2010, she joined IVHM Centre, Cranfield University as a doctoral researcher working on prognostics and health management of aircraft avionics.

Her main interests include health monitoring and prognostics.

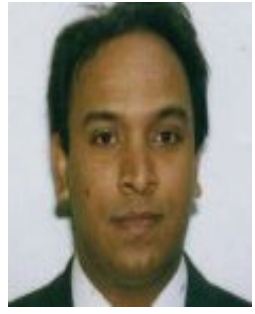

Suresh Perinpanayagam (M'12) is a Lecturer and Head of Electronic Prognostics (ePHM Group) at the Integrated Vehicle Health Management (IVHM) Centre at Cranfield University. Suresh has rapidly established ePHM group has obtained grants amounting to $£ 1.68 \mathrm{M}$ in total from industrial, EPSRC, TSB and EU-FP7 projects. Suresh has published 50 peer-reviewed journal/conference papers. Suresh is a member of the working group developing the IEEE Standard for Prognostics and Health Management. He has published one book chapter on 'Sensors, Instrumentation and Signal Processing' in the SAE International book on Integrated Vehicle Health Management and numerous research papers.

Suresh is an associate editor for IEEE Transactions on Power Electronics Special Issue on Robust Design and Reliability in Power Electronics. He is a member of the Editorial Board of the Microelectronics Reliability Special Issue on Reliability of Power Electronics.

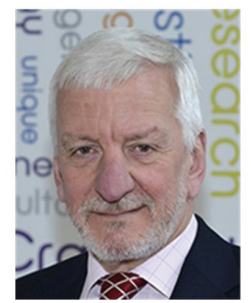

Ian Jennions's career spans some 40 years, working mostly for a variety of gas turbine companies. He has a Mechanical Engineering degree and $\mathrm{ahD}$ in $\mathrm{CFD}$ both from Imperial College, London. He has worked for Rolls-Royce (twice), General Electric and Alstom in a number of technical roles, gaining experience in aerodynamics, heat transfer, fluid systems, mechanical design, combustion, services and IVHM.

He moved to Cranfield in July 2008 as Professor and Director of the newly formed IVHM Centre. The Centre is funded by a number of industrial companies, including Boeing, BAE Systems, Thales, Meggitt, MOD, DRS and Alstom Transport. He has led the development and growth of the Centre, in research and education, since its inception. 
2016-02-25

\section{Aging detection capability for switch-mode power converters}

Kaur Mann, Jaspreet

Institute of Electrical and Electronics Engineers

Jaspreet Kaur Mann, Suresh Perinpanayagam and lan Jennions. Aging detection capability for switch-mode power converters. IEEE Transactions on Industrial Electronics, Volume:63, Issue:5, pp3216 - 3227

http://dx.doi.org/10.1109/TIE.2016.2535104

Downloaded from Cranfield Library Services E-Repository 